reviewers independently screened titles using Covidence software, extracted data in standardised forms, and conducted methodological quality appraisal (using the Critical Appraisal Skills Programme checklist). A thematic synthesis of included studies was conducted, using line by line coding, developing descriptive and analytical themes using NVivo 12 software. The GRADE-CERQual (Confidence in the Evidence from Reviews of Qualitative research) approach was used to summarise the certainty of the evidence.

Results Forty-seven papers, describing 42 unique datasets, incorporating data from 710 patients were included. The study recruited from a mix of inpatient, outpatient, hospice and community settings across 12 different countries with $49 \%$ of studies conducted in the US. Preliminary findings demonstrate spirituality was expressed both through religious practices, and through relationships or connectedness with family, friends, nature and with a sense of self. Spirituality has on-going importance to those at the end of life as a means of finding hope, meaning and certainty, living remaining life well and adjusting to dying. Considering the broad sense of spirituality expressed by participants, practice improvements may include healthcare providers acknowledging and supporting patients' desires for creating meaning, purpose and connectedness through listening, connecting, and engaging in discussions about these needs. Greater access to chaplains for participants who expressed spirituality through religion may also be of benefit.

Conclusions Qualitative research is increasingly used in the development of evidence-based health care and clinical guidelines. Such methodologies provide deeper insights into patient experiences of healthcare services and ill-health and support the provision of healthcare that is more patient-centred. The findings from this systematic review highlight the importance of spirituality for patients at the end of life as means of finding hope, meaning and certainty, living remaining life well and adjusting to dying. These findings will be used to inform the development of a national clinical guideline for care of the dying adult in the Republic of Ireland.

PROSPERO: The protocol for this systematic review was registered on PROSPERO, CRD42019122062

\section{DESIGN, METHODS, AND REPORTING OF STUDIES EVALUATING IMPACT OF CARDIOVASCULAR CLINICAL PREDICTION RULES: A SYSTEMATIC REVIEW}

Jong-Wook Ban. Evidence-Based Health Care Programme, University of Oxford, Oxford, UK

\subsection{6/bmjebm-2019-EBMLive.23}

Objectives Clinical prediction rules (CPRs) are tools that help clinicians address diagnostic or prognostic uncertainties using symptoms, signs, and test results of a patient. Developing a CPR involves three steps: derivation, validation, and impact study. Poor design, methods, or reporting in these steps can lead to research waste. No study has evaluated whether impact studies are conducted with appropriate design, methods, and reporting. Therefore, we conducted a systematic review to summarize design, methods, and reporting of impact studies of cardiovascular CPRs. We also compared quality of methods and reporting of appropriately designed impact studies with that of control studies evaluating other types of nonpharmacologic intervention.
Method We reviewed studies evaluating the impact of cardiovascular CPRs included in the International Register of CPRs. We identified impact studies by conducting forward citation searches of these CPRs. For some cardiovascular CPRs never published in a journal, we searched electronic databases to identify their impact studies. We categorized the design of impact studies as ideal (randomized experiment), alternative (non-randomized experiment excluding uncontrolled beforeafter study), and inappropriate (all other study designs). For impact studies with appropriate study design, we assessed their methods using the Cochrane risk of bias and ROBINS-I tools. We assessed their report using the CONSORT statement, relevant extensions to the CONSORT statement, and TREND statement. For each impact study with appropriate design, we identified a contemporaneous control study with matching design published in the same journal and compared their methods and reporting.

Results We screened a total of 42769 references, 40644 from forward citation searches of 194 CPRs and 2125 from electronic database searches of 4 CPRs. Of 110 of impact studies of cardiovascular CPRs found, 59.1\% used inappropriate designs (40 uncontrolled before-after studies, 7 cohort studies, and 18 non-comparative studies), $9.1 \%$ used alternative designs ( 2 non-randomized trials, 4 interrupted time series studies, and 4 repeated measures studies), and 31.8\% used ideal designs (12 cluster randomized trials and 23 randomized controlled trials). Overall risk of bias was substantial in $80 \%$ (31 of 45) of impact studies. Mean proportion of domains from reporting guidelines that impact studies adhered to was only $44.1 \% \quad$ (9.3 of 21). There was no clear difference between impact and matched control studies in the proportion of studies with substantial risk of bias and the mean proportion of reporting domains studies did not comply with.

Conclusions We conducted the first systematic review that evaluated design, methods, and reporting of impact studies. We found the vast majority of impact studies either used study designs inappropriate for assessing the effect of using CPRs, applied methods carrying substantial risk of bias, or did not comply with reporting guidelines. All stakeholders of CPR development should take concerted actions to increase the value of research.

\section{ADDING CAPACITY: GETTING EBP INTO THE CURRICULUM FOR ALL HEALTH PROFESSIONALS IN IRELAND}

${ }^{1}$ Niamh O'Rourke, ${ }^{2} \mathrm{E}$ Lehane, ${ }^{2} \mathrm{H}$ Agreli, ${ }^{2} \mathrm{~S} O \mathrm{O}^{\prime} \mathrm{Connor},{ }^{2} \mathrm{~J}$ Hegarty, ${ }^{2} \mathrm{P}$ Leahy-Warren, ${ }^{2} \mathrm{D}$ Bennet, ${ }^{2} \mathrm{C}$ Blake, ${ }^{2} \mathrm{~F}$ Burke, ${ }^{2} \mathrm{M}$ Corrigan, ${ }^{2} \mathrm{~J}$ Drennan, ${ }^{2} \mathrm{M}$ Hayes, ${ }^{2} \mathrm{E}$ Heffernan, ${ }^{2} \mathrm{~F}$ Horgan, ${ }^{2} \mathrm{H}$ Lynch, ${ }^{2} \mathrm{~J} \mathrm{Mc}$ eigh, ${ }^{2} \mathrm{~N}$ Müller, ${ }^{2} \mathrm{E} O \mathrm{O}^{\mathrm{K}} \mathrm{Keeffe},{ }^{2} \mathrm{C} \mathrm{O}$ T Tuathaigh, ${ }^{2} \mathrm{~L}$ Sahm, ${ }^{3} \mathrm{E}$ O'Toole, ${ }^{2} \mathrm{E}$ Savage. 'Department of Health, Dublin, Ireland; ${ }^{2}$ UCC, Cork, Ireland; ${ }^{3} \mathrm{HSE}$, Dublin, Ireland

\subsection{6/bmjebm-2019-EBMLive.24}

Objectives Evidence Based Practice (EBP) is a cornerstone of delivering clinically effective care. An EBP capacity-building project commenced in Ireland in 2016, to build capability and leadership for EBP, with the ultimate goal of improving patient outcomes. A key part of this project was the development of a competency framework for education in evidencebased practice, to ensure responsiveness of education standards and curricula of healthcare professionals in this area. 
- Develop a competency framework for healthcare professionals in EBP and clinical effectiveness education.

- Inform academic curricula and professional development programmes at third level.

- Provide a guide for attainment of knowledge, skills and attributes/behaviours for health service staff.

- Dissemination of the competency framework to Irish statutory health regulators and educators, to inform professional standards for registration courses and continuous professional development of healthcare professionals in Ireland.

\section{Method Multiyear project (2016-2019):}

- Baseline research on the teaching of evidence-based practice in Ireland; literature review, interviews with international experts and national survey of EBP teaching at third-level institutions

- Two national consultation events on EBP education in Ireland with regulators, researchers and educators

- Analysis of health regulators requirements in Ireland in relation to education and professional standards

- Development of a competency framework by UCC; documentary analysis of national and international documents pertaining to EBP and clinical effectiveness education; relevant competencies, curriculum considerations, teaching \& learning methods and assessment strategies

- Stakeholder consultation; 13 focus groups with representatives from the health services, clinical practice, third level education \& professional training sectors, regulator/accrediting bodies, and the Department of Health, to examine draft competency framework

- Policy makers in the Department of Health, health service providers and the regulators of health professionals (medicine, nursing and allied health professionals) convened an implementation group to oversee dissemination and implementation.

Results Evidence-based practice is a core component of clinical effectiveness. Additional competencies in quality improvement processes and implementation science strategies were deemed essential for patient outcomes. Professional practice competencies which addressed the need for effective communication, collaborative practice and leadership were specifically recommended.

An overarching inter-disciplinary competency framework was proposed for clinical effectiveness education, which included the following domains: Evidence-based practice; Quality improvement processes; Implementation strategies and Professional practice.

Stakeholders recommended the explicit integration of EBP competencies throughout academic and clinical learning domains of health professional curricula.

Teaching methods recommended were based on an active learning approach and included: group-based projects; roleplay/modelling; simulations and case-based studies that aid learners to apply and relate theory to real-world practice. Conclusions A competency framework for clinical effectiveness education for health and social care professionals was published by the Department of Health in Ireland in 2018. It is intended that this framework will provide guidance to healthcare educators and regulators in the blueprinting of curricula, learning outcomes, assessment strategies, and graduate/clinician attributes.

By means of consultation with stakeholders and working in collaboration with regulators and policy makers, we can translate evidence into policy and practice, to improve patient outcomes.

Through inclusion of evidence-based practice in the curricula of all healthcare professionals at both undergraduate and post-graduate level, we aim to build capacity, educate health professionals to make informed choices, enhance real-world practice and encourage the next generation of leaders in evidence-based healthcare in Ireland.

\section{EBM+: INCREASING THE SYSTEMATIC USE OF MECHANISTIC EVIDENCE}

Jon Williamson. University of Kent, Canterbury, UK

\subsection{6/bmjebm-2019-EBMLive.25}

Objectives The EBM+ programme seeks to ensure that existing evidence of mechanisms is systematically evaluated when assessing the effectiveness of an intervention or the effects of an exposure. Basing assessments on mechanistic studies in addition to clinical studies leads to more reliable assessments and can sometimes speed up the time taken to make decisions.

The objectives of this paper are to:

1. Describe the aims of the EBM+ programme;

2. Explain the rationale behind the EBM+ programme;

3. Describe EBM+ methods for systematically assessing mechanistic evidence;

4. Discuss the feasibility of the EBM+ approach.

Method Objectives 1-3 of the paper will be met by discussing the EBM+ programme as set out by Parkkinen et al. (2018), Evaluating evidence of mechanisms in medicine, Springer, https:/link.springer.com/book/10.1007/978-3-319-94610-8

The EBM+ programme arose from recent philosophical work on the epistemology of causality. Objectives 1 and 2 will be met by explaining what this work is, why it is new and interesting, and how it could lead to more systematic use of existing evidence in medicine and public health.

Objective 3, an account of the EBM + methods for assessing mechanistic evidence, will be met by providing an overview of the methods set out in Parts II and III of Parkkinen et al. (2018), which cover (i) gathering evidence of mechanisms; (ii) evaluating evidence of mechanisms; and (iii) using evidence of mechanisms to evaluate efficacy and external validity.

Objective 4 will be met by providing a new feasibility analysis of EBM+.

Results The results of the feasibility analysis include:

- A critical analysis of four different grounds for thinking the $\mathrm{EBM}+$ approach might be feasible to implement in practice. Two of these lines of argument are shown to fail, while two are shown to provide good evidence for the feasibility of $\mathrm{EBM}+$.

- A critical analysis of the objection that the EBM+ approach is prone to bias and subjectivity. Four responses to that objection are considered, with one shown to fail and three to be successful. 